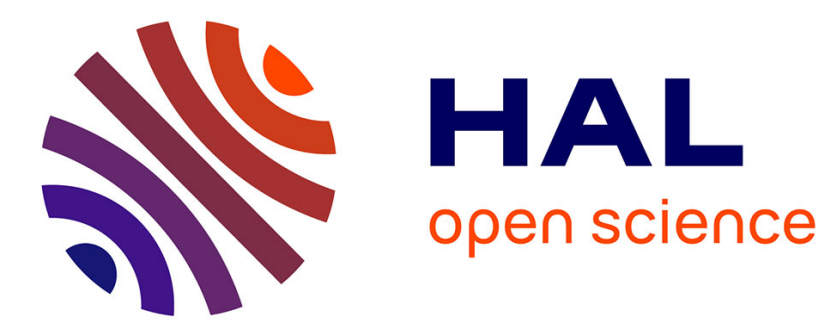

\title{
Reactor Neutrinos
}

\author{
T. Lasserre, H. W. Sobel
}

\section{To cite this version:}

T. Lasserre, H. W. Sobel. Reactor Neutrinos. Comptes Rendus. Physique, 2005, 6, pp.749-757. 10.1016/j.crhy.2005.08.002 . hal-00016511

\section{HAL Id: hal-00016511 https://hal.science/hal-00016511}

Submitted on 5 Jan 2006

HAL is a multi-disciplinary open access archive for the deposit and dissemination of scientific research documents, whether they are published or not. The documents may come from teaching and research institutions in France or abroad, or from public or private research centers.
L'archive ouverte pluridisciplinaire HAL, est destinée au dépôt et à la diffusion de documents scientifiques de niveau recherche, publiés ou non, émanant des établissements d'enseignement et de recherche français ou étrangers, des laboratoires publics ou privés. 


\title{
Reactor Neutrinos
}

\author{
Thierry Lasserre ${ }^{\mathrm{a}}$, Henry W. Sobel ${ }^{\mathrm{b}}$ \\ ${ }^{a}$ DAPNIA/SPP, CEA/Saclay, 91191 Gif-sur-Yvette, France E Laboratoire Astroparticule et Cosmologie (APC), 11, place \\ Marcelin Berthelot, 75231 Paris cedex 05, France \\ ${ }^{\mathrm{b}}$ Department of Physics and Astronomy, 4129 Frederick Reines Hall, University of California, Irvine, CA 92697-4575, USA
}

\begin{abstract}
We review the status and the results of reactor neutrino experiments, that toe the cutting edge of neutrino research. Short baseline experiments have provided the measurement of the reactor neutrino spectrum, and are still searching for important phenomena such as the neutrino magnetic moment. They could open the door to the measurement of coherent neutrino scattering in a near future. Middle and long baseline oscillation experiments at Chooz and KamLAND have played a relevant role in neutrino oscillation physics in the last years. It is now widely accepted that a new middle baseline disappearance reactor neutrino experiment with multiple detectors could provide a clean measurement of the last undetermined neutrino mixing angle $\theta_{13}$. We conclude by opening on possible use of neutrinos for Society : Non Proliferation of Nuclear materials and Geophysics. To cite this article: Th. Lasserre, H.W. Sobel, C. R. Physique 6 (2005).
\end{abstract}

\section{Résumé}

\section{Neutrinos auprès des réacteurs.}

Les expériences de détection des (anti)neutrinos issus des réacteurs nucléaires ont joué un rôle déterminant en physique des neutrinos depuis leur découverte. Les expériences au plus près du cœur nucléaire ont permis non seulement de comprendre la source d'antineutrinos, mais également de contraindre certaines propriétés importantes comme le moment magnétique du neutrino. Une expérience très proche du cœur pourrait mettre en évidence la diffusion cohérente des neutrinos. Les expériences à moyenne ou longue portées, Chooz et KamLAND, ont joué ces dernières années un rôle fondamental dans la compréhension de l'oscillations des neutrinos. De nouvelles expériences sont en préparation pour mesurer le dernier angle de mélange encore inconnu, $\theta_{13}$, en utilisant des techniques similaires mais plus précises. Nous concluons en décrivant deux applications possibles : l'utilisation de détecteurs d'antineutrinos pour contribuer à la non prolifération des matières nucléaires fissiles, et l'étude de la géophysique à l'aide des antineutrinos "terrestres". Pour citer cet article: Th. Lasserre, H.W. Sobel, C. R. Physique 6 (2005).

Key words: Neutrinos; Nuclear Reactor ; Keyword3

Mots-clés : Neutrinos; Réacteur nucléaire; Mot-clé3

Email addresses: tlasserr@cea.fr (Thierry Lasserre), hsobel@uci.edu (Henry W. Sobel). 


\section{Neutrino discovery}

Invented by Pauli [1] in 1930, named by Fermi in 1934 and later modeled in his theory of beta decay [2], the extraordinarily weakly coupling neutrino was first searched for by Reines and Cowan. Starting at the Hanford nuclear reactor (Washington) they later moved to the new Savannah River Plant (South Carolina) to perform their definitive and ground-breaking experimental detection. This feat had two consequences: resolving and clarifying the highly unsatisfactory situation of a fundamental particle needed for the consistency of theory, but virtually unobservable, and demonstrating the possibility of doing "neutrino physics". This opened the door to the use of neutrinos as a sensitive probe of particle physics. Indeed, several years after the completion of the seminal work of Reines and Cowan, neutrinos were beginning to be used regularly to investigate the weak interactions, the structure of nucleons and the properties of their constituent quarks.

In the first crude experiment of 1953 [3], their goal was to demonstrate unambiguously a reaction caused in a target by a neutrino produced elsewhere. The experiment pioneered the delayed coincidence technique to search for the reaction: $\overline{\nu_{e}}+p \rightarrow e^{+}+n$ where an electron antineutrino from the Hanford nuclear reactor interacted with a free proton in a large tank filled with cadmium loaded liquid scintillator. The positron and the resultant annihilation gamma-rays are detected as a prompt signal while the neutron is thermalized in the liquid scintillator and subsequently captured by the cadmium. The excited nucleus then emits gamma radiation which is detected as the delayed signal. The first result, at two standard deviations, was followed in 1956 and 1958 by more precise experiments $[4,5,6]$ where the significance improved to over four standard deviations. In addition to the detection, the reaction cross-section was measured to be $11 \pm 2.6 \times 10^{-44} \mathrm{~cm}^{2}$ [6]. Nowadays, reactor neutrinos are still detected through similar experimental methods.

\section{Neutrinos from reactors}

Fission reactors are prodigious producers of neutrinos (about $10^{20} \overline{\nu_{e}} s^{-1}$ per nuclear core). The fissioning of ${ }^{235} \mathrm{U}$ produces elements which must shed neutrons to approach the line of stability. The beta decays of this excess produce approximately six electron antineutrinos per fission. In modern reactors, the uranium fuel is enriched to a few percent in ${ }^{235} \mathrm{U}$, but there are also significant contributions to the neutrino flux from the fissioning of ${ }^{238} \mathrm{U},{ }^{239} \mathrm{Pu}$, and ${ }^{241} \mathrm{Pu}$. During a typical fuel cycle, the $\mathrm{Pu}$ concentrations increase so the neutrino flux from ${ }^{239} \mathrm{Pu}$, and ${ }^{241} \mathrm{Pu}$ grows with time (see Figure 1). The $\overline{\nu_{e}}$ spectrum is calculated from measurements of the beta decay spectra of ${ }^{235} \mathrm{U},{ }^{239} \mathrm{Pu}$, and ${ }^{241} \mathrm{Pu}[7]$ after fissioning by thermal neutrons. Since ${ }^{238} \mathrm{U}$ fissions with fast neutrons this technique cannot be used. In this case, a summation of the $\overline{\nu_{e}}$ from all possible beta decay processes is performed. Since ${ }^{238} \mathrm{U}$ contributes only about about $11 \%$ to the neutrino signal, and further since the error associated with this summation method is less than $10 \%$ it contributes less than $1 \%$ to the overall uncertainty in the $\overline{\nu_{e}}$ flux. Experimentally, the observed rate of positron production from $\overline{\nu_{e}}+p \rightarrow e^{+}+n$ has been compared to the predicted rate in order to test the precision of the $\overline{\nu_{e}}$ spectra prediction [8].
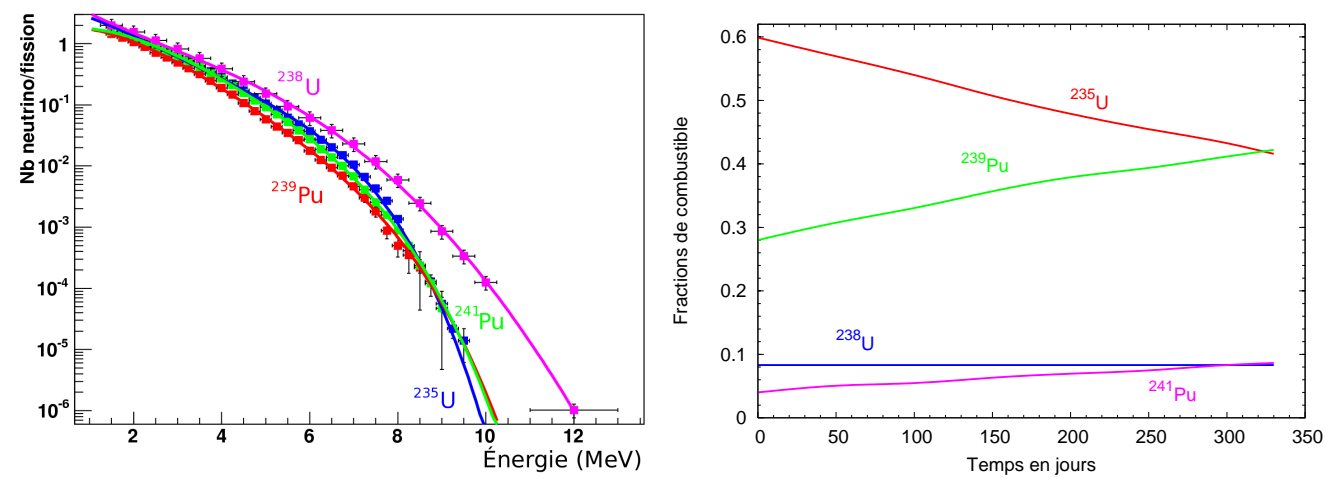

Figure 1. $\overline{\nu_{e}}$ spectra of the four dominant fissioning isotopes (left). Percentage of fissions of the main fissile elements during a fuel cycle (right). 


\section{Elastic scattering}

In the Standard Model, the differential cross section for the purely leptonic (weak scattering) elastic scattering reaction, $\nu_{e}+e \rightarrow \nu_{e}+e$, is given by:

$$
\left(\frac{d \sigma}{d T}\right)_{W K}=C\left[g_{L}^{2}+g_{R}^{2}\left(1-\frac{T}{E_{\nu}}\right)^{2}-g_{L} g_{R} \frac{m_{e} T}{E_{\nu}^{2}}\right]
$$

where $C=2 G_{F}^{2} m_{e} / \pi, g_{L}=\sin ^{2} \theta_{W}+1 / 2$ for $\nu_{e}, g_{L}=\sin ^{2} \theta_{W}-1 / 2$ for $\nu_{\mu}$ and $\nu_{\tau}$, and $g_{R}=\sin ^{2} \theta_{W}\left(\theta_{W}\right.$ is the Weinberg angle). $T=E_{e}-m_{e}$ is the kinetic energy of the recoil electrons. The first experiment to detect this reaction [9] took advantage of a well shielded location only 11 meters from the center of the Savannah River reactor. The intense flux of $2.2 \times 10^{13} \nu_{e} \mathrm{~cm}^{-2} \mathrm{sec}^{-1}$ and the regular up/down cycling of the reactor gave a reasonable signal in a small target and eliminated non-reactor-associated backgrounds. The experimental cross section was measured with a precision of $\pm 26 \%$. Analysis of the experimental results also produced a crude $( \pm 17 \%)$ measurement of $\theta_{W}$.

\section{Coherent neutrino scattering}

If the neutrino energy is small, the neutrino wavelength can be large when compared to the size of a nucleus. In this case, the neutrinos can scatter coherently from the nucleons and the scattering cross-section would be proportional to the square of the weak nuclear charge [10]:

$$
\frac{d \sigma}{d(\cos \theta)}=\frac{G^{2}}{8 \pi}\left[Z\left(4 \sin ^{2} \theta_{W}-1\right)+N\right]^{2} E^{2}(1+\cos \theta) .
$$

In 1977, Freedman et. al. [10] pointed out that the large cross-section, particularly important for supernova dynamics, should be experimentally measured at nuclear reactors. Drukier and Stodolsky [11] suggested using cryogenic bolometers to observe the very low energy nuclear recoil (100's of eV's for MeV neutrinos), but that technique has not been successful up to now.

\section{Neutrino magnetic moment}

In the Standard Model, the massless neutrinos do not have magnetic moments, but this is not the case if neutrinos do have mass [12]. However, introducing this effect in the model leads to moments of at least eight orders of magnitude below currently accessible experimental limits. These limits are derived from reactor $\bar{\nu}_{e}$ 's $[9,13,14,15]$, in the range of $(0.9-4) \times 10^{-10} \mu_{B}$, where $\mu_{B}$ is the Bohr magneton. Various astrophysical observations also yield limits on the neutrino magnetic moment in the range from $10^{-12} \mu_{B}$ to $4 \times 10^{-10} \mu_{B}$ [16]. Therefore, a positive observation of such large magnetic moments would imply additional physics beyond the Standard Model. Experimentally, neutrino-electron scattering has been used in the search for neutrino magnetic moments. If $\mu_{\nu} \neq 0$, the differential cross section of neutrinon-electron scattering is an incoherent sum of weak scattering and magnetic scattering $[17]$ :

$$
\left(\frac{d \sigma}{d T}\right)_{E M}=\mu_{\nu}^{2} \frac{\pi \alpha_{e m}^{2}}{m_{e}^{2}}\left(\frac{1}{T}-\frac{1}{E_{\nu}}\right)
$$

where $\mu_{\nu}$ is in units of $\mu_{B}, E_{\nu}$ is the neutrino energy, $T=E_{e}-m_{e}$, and $T\left(E_{e}\right)$ is the kinetic (total) energy of the recoil electrons.

The contribution from electromagnetic scattering increases rapidly at lower energies, so the signature of a non-zero neutrino magnetic moment would be an enhancement of the event rate at lower energies. The current constraint including the latest KamLAND data results in a limit on the neutrino magnetic moment at $90 \%$ C.L. of $\mu_{\nu} \leq 1.1 \times 10^{-10} \mu_{B}$ with the limit at $\Delta m^{2}=6.6 \times 10^{-5} \mathrm{eV}^{2}$ and $\tan ^{2} \theta=0.48$. This result is comparable to the most recent magnetic moment limits from reactor neutrino experiments of $1.3 \times 10^{-10} \mu_{B}$ (TEXONO) [14] and $1.0 \times 10^{-10} \mu_{B}(\mathrm{MUNU})[15]$, albeit for neutrinos and not antineutrinos. 


\section{Neutrino Oscillations}

There is now convincing evidence for flavor conversion of atmospheric [18], solar [19], reactor and accelerator neutrinos $[20,21,22,23,24,25]$. Thus, neutrinos do have masses, and neutrino oscillation is the most promising scenario to explain the data (see [26] for details on the neutrino oscillation mechanism). Reactor neutrino experiments measure the survival probability $P\left(\overline{\nu_{e}} \rightarrow \overline{\nu_{e}}\right)$ of the $\overline{\nu_{e}}$ emitted by nuclear power stations at a given distance $(\mathrm{L})$. This disappearance probability does not depend on the Dirac CP phase $\delta$. Furthermore, thanks to the combination of the $\mathrm{MeV}$ range neutrino energies (E) and the short baselines (less than thousand kilometers) the modification of the oscillation probability induced by the coherent forward scattering from matter electrons (so-called matter effect) can be neglected in first approximation. If neutrinos masses satisfy $m_{1}<m_{2}<m_{3}$ (so-called "Normal Hierarchy", NH), the survival probability can be written:

$$
\begin{aligned}
1-P\left(\overline{\nu_{e}} \rightarrow \overline{\nu_{e}}\right. & =4 \sin ^{2} \theta_{13} \cos ^{2} \theta_{13} \sin ^{2} \frac{\Delta m_{31}^{2} L}{4 E}+\cos ^{4} \theta_{13} \sin ^{2}\left(2 \theta_{12}\right) \sin ^{2} \frac{\Delta m_{21}^{2} L}{4 E} \\
-2 & \sin ^{2} \theta_{13} \cos ^{2} \theta_{13} \sin ^{2} \theta_{12}\left(\cos \frac{\left(\Delta m_{31}^{2}-\Delta m_{21}^{2}\right) L}{2 E}-\cos \frac{\Delta m_{31}^{2} L}{2 E}\right) .
\end{aligned}
$$

The first two terms of the right side of Equation 1 are, respectively, the atmospheric $\left(\Delta m_{31}^{2}=\Delta m_{a t m}^{2}\right)$ and solar driven $\left(\Delta m_{21}^{2}=\Delta m_{\text {sol }}^{2}\right)$ oscillations, while the third term is an interference between both contributions [27]. An experiment is only sensitive to the values of $\Delta m^{2}$ such that $L>L_{\mathrm{OSc}}($ meter $)=2.48 E(M e V) / \Delta m^{2}\left(e V^{2}\right)$. We notice here that $\theta_{13}$ is the mixing angle that couples the heaviest neutrino field to the electron field (NH). If $\Delta m_{\text {sol }}^{2}<<\Delta m_{a t m}^{2}$ and/or $\theta_{13}$ is small enough, the solar driven and the atmospheric driven neutrino oscillations decouple. The mixing is then radically simplified, leading to two neutrino mixing formula:

$$
1-P\left(\overline{\nu_{e}} \rightarrow \overline{\nu_{e}}\right)=\sin ^{2} 2 \theta_{i} \cdot \sin ^{2}\left(1.27 \frac{\Delta m_{i}^{2}\left[e V^{2}\right] L[m]}{E_{\overline{\nu_{e}}}[\mathrm{MeV}]}\right)
$$

For the reactor neutrino oscillations we can consider two extreme cases: $\Delta m_{i}^{2}=\Delta m_{21}^{2}$ and $\theta_{i} \sim \theta_{\text {sol }}$ if the baseline considered exceeds a few tens of kilometers, and $\Delta m_{i}^{2}=\Delta m_{31}^{2}$ and $\theta_{i}=\theta_{\text {atm }}$ if it is does not exceed a few kilometers.

\subsection{The pioneering experiments}

In the eighties and nineties, several experiments [28,29,30,31] were performed at a few ten's of meters from nuclear reactor cores at Goesgen (Switzerland), Rovno, Krasnoyarsk (Russia), and ILL Grenoble, Bugey (France). Since the knowledge of the neutrino source was not better than $10 \%$, they compared the neutrino rate at different distances to improve their sensitivity. The most stringent bounds on the oscillation parameters of this generation of experiments were obtained at Bugey. The $\overline{\nu_{e}}$ spectra were measured at three different source-detector distances $(15,40$, and $95 \mathrm{~m})$, using three identical modules filled by ${ }^{6} \mathrm{Li}$-doped liquid scintillator. Measurements were in agreement with the no-oscillation expectation, constraining the oscillation parameters in the region $\Delta m_{\text {atm }}^{2} \sim$ $10^{-2} \mathrm{eV}^{2}$ [31]. From this set of experiments (see Figure 2), the absolute normalization and the spectral shape of reactor $\overline{\nu_{e}}$ are known to a precision of about $2 \%$ [32].

\subsection{Exploring the "atmospheric oscillation"}

In the fall of the nineties, two experiments were performed to test the hypothesis that neutrino oscillations occur in the parameter region probed by the atmospheric neutrino experiments, $\Delta m_{a t m}^{2} \sim 10^{-3} \mathrm{eV}^{2}[20]$.

The Chooz experiment was located in the Ardennes region of France, $1050 \mathrm{~m}$ away from the double unit Chooz nuclear reactors $\left(\mathrm{PWR}, 8.4 \mathrm{GW}_{\mathrm{th}}\right)$. The detector was located in an underground laboratory below a $100 \mathrm{~m}$ rock overburden (300 $\mathrm{m}$ of water equivalent, mwe), providing, for the first time at reactors, a strong reduction of the cosmic ray induced backgrounds. The homogeneous detector was filled by a 5 ton Gd-doped liquid scintillator target, surrounded by a thick active (scintillating) buffer and a muon veto. The external tank was surrounded by an additional layer of low radioactive sand. This composition of shielding moderates neutrons induced by muons outside of the detector as well as the $\gamma$ 's produced by the rocks. Since the two Chooz reactors were commissioned after the start of the experiment, there was a unique opportunity to perform an in-situ background measurement. 

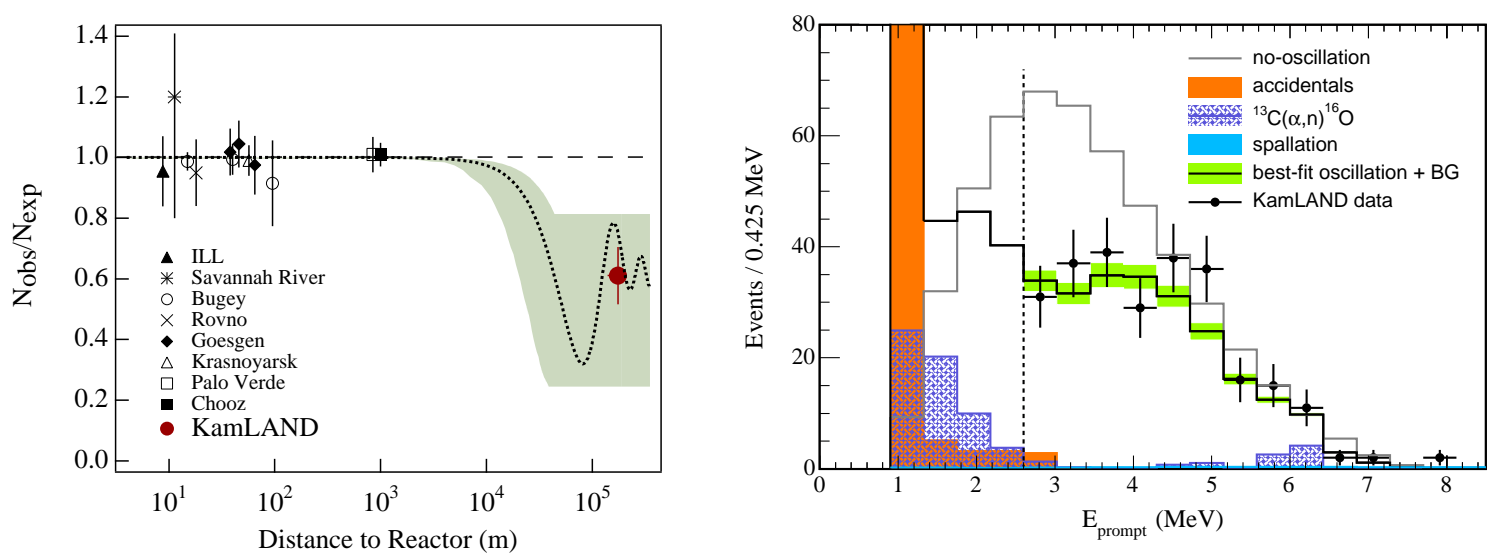

Figure 2. (left) Ratio of the observed number of neutrino events with respect to the no-oscillation case for all reactor neutrino experiments. The source-detector distances are displayed on the x-axis. Among all experiments, only KamLAND has observed a disappearance thanks to its very long source-detector average distance [24]. (right) Measurement of neutrino oscillation with KamLAND, and the evidence of spectral distortion. The energy spectrum (visible energy in the detector) is displayed together with best-fit oscillation spectrum (in solid black). The unoscillated spectrum (grey histogram), and the other backgrounds (accidental, spallation, and $\alpha$ induced) are also shown [34].

The Palo Verde experiment was located in an underground bunker under 12 meters of rock (32 mwe), 750 and 890 meters away from a 3 -unit nuclear power station $\left(11.6 \mathrm{GW}_{\mathrm{th}}\right)$ in the Arizona desert. The low overburden required the use of a segmented detector to reduce the background. It was composed of 66 acrylic cells of 9 meters filled with a Gd-doped liquid scintillator, surrounded by a 1 meter thick water shielding and an efficient liquid scintillator muon veto.

Neither Chooz nor Palo Verde observed any evidence of neutrino oscillation. The results could be presented as the energy averaged ratio $(\mathrm{R})$ between $\overline{\nu_{e}}$ detected and expected $R_{C h o o z}=1.01 \pm 2.8 \%$ (stat.) $\pm 2.7 \%$ (syst.) and $R_{\text {Paloverde }}=1.01 \pm 2.4 \%$ (stat.) $\pm 5.1 \%$ (syst.). Both experiments excluded any $\overline{\nu_{e}} \rightarrow \overline{\nu_{x}}$ oscillation driven by $\Delta m_{\text {atm }}^{2} \sim 10^{-3} \mathrm{eV}^{2}$, except for small mixing. Assuming the conservation of CPT, they excluded the $\nu_{\mu} \rightarrow \nu_{e}$ oscillation solution in the Kamiokande experiment [20]. The Chooz experiment still provides the world best constraint on the $\theta_{13}$ mixing angle: $\sin ^{2}\left(2 \theta_{13}\right)<0.14$, at $\Delta m_{a t m}^{2}=2.510^{-3} \mathrm{eV}^{2}[33]$.

\subsection{Exploring the "solar oscillation"}

A reactor neutrino experiment with a baseline distance of hundreds of kilometers is sensitive to the Large Mixing Angle (LMA) oscillation solution of the solar electron neutrino deficit (see [19]). If the reactor-detector distance is slightly larger than the oscillation length, neutrino oscillations are observable as an integral reduction of the interaction rate, as well as a periodic modulation of the $\overline{\nu_{e}}$ spectrum, which provides a sensitivity to $\Delta m_{\text {sol }}^{2}$.

The KamLAND experiment [24] is located at the site of the earlier Kamiokande in the Kamioka mine (Japan), below 2700 mwe of rock. The detector consists of 1 kton of ultra-pure liquid scintillator contained in a $13 \mathrm{~m}$ diameter transparent nylon balloon suspended in a non-scintillating oil buffer. The balloon is surrounded by about 1900 PMTs mounted on a $18 \mathrm{~m}$ diameter stainless steel vessel. KamLAND is surrounded by more than 50 nuclear power units, at an averaged distance of $180 \mathrm{~km}$. KamLAND has published data from 766 ton-year of exposure. In the absence of neutrino oscillation 365.2 events are expected (above $2.6 \mathrm{MeV}$ to eliminate the geo-neutrinos background, see section 7.2), but only $258 \overline{\nu_{e}}$ candidate events have been detected. Accounting for 18 expected background events, the statistical significance for reactor $\overline{\nu_{e}}$ disappearance is $99.998 \%$. Assuming CPT invariance, this result excludes all but the LMA solution to the solar neutrino deficit. It suggests that solar neutrino flavor transformation through the MSW matter effect (see [19]) has a direct correspondence to antineutrino oscillations in vacuum. In addition, the energy spectrum measured by KamLAND disagrees with the expected spectral shape in the absence of neutrino oscillation at $99.6 \%$ significance and prefers the distortion expected from $\overline{\nu_{e}}$ oscillation effect (see Figure 2). A two-neutrino oscillation analysis of the KamLAND data gives $\Delta m_{\text {sol }}^{2}=7.9_{-0.5}^{+0.6} \times 10^{-5} \mathrm{eV}^{2}[34]$.

The Borexino experiment in Italy is designed to detect low-energy solar neutrinos via electron scattering, using 0.3 kton of ultra-pure liquid scintillator target, observed by 2200 PMTs [19]. Borexino is surrounded by many 
nuclear power stations, but with a characteristic distance of $800 \mathrm{~km}$ (Italy does not host nuclear power plants). In this case, the shape of the neutrino spectrum will appear unchanged since the baseline is too large. Thus, only lower limits on $\Delta m_{\text {sol }}^{2}$ can be derived after a few years of data taking, since about 30 events are expected each year in the no-oscillation case [35].

In the future, a new reactor neutrino experiment with a baseline corresponding to the first oscillation dip (about $60 \mathrm{~km}$ ) could provide a high precision determination of $\sin ^{2} \theta_{12}$. With an exposure of $60 \mathrm{GW}_{\mathrm{th}} \mathrm{kton}$ year and a systematic error of $2 \%, \sin ^{2} \theta_{12}$ could be determined with an uncertainty of $2 \%$ at one standard deviation [36].

\subsection{Measuring the third and last neutrino mixing angle $\theta_{13}$}

Considering only the three known families, the neutrino mixing matrix is parametrized by three mixing angles. The angle $\theta_{12}$ has been measured to be large, $\sin ^{2}\left(2 \theta_{12}\right) \sim 0.8$, by the combination of the solar neutrino experiments and KamLAND (see [19]). The angle $\theta_{23}$ has been measured to be close to maximum, $\sin ^{2}\left(2 \theta_{23}\right)>0.9$, by atmospheric neutrino experiments $[20,18]$ as well as the long baseline accelerator neutrino experiment K2K [25]. However, we only have an upper limit to the mixing angle $\theta_{13}$, given mainly by the Chooz experiment, $\sin ^{2}\left(2 \theta_{13}\right)<$ 0.2. The large value of both $\theta_{12}$ and $\theta_{23}$ indicates a strong difference between leptonic and quark mixings, whereas the smallness of $\theta_{13}$ testifies to the peculiarity of the neutrino sector. The value of $\theta_{13}$ is not only of fundamental interest to understand leptonic mixing, but it is also necessary to plan for the future experimental program in neutrino physics, since CP-violating effects are proportional to $\sin ^{2} \theta_{13}$.

New accelerator neutrino beams coupled with off-axis detectors, will search for a $\nu_{e}$ appearance signal. The observation of a $\nu_{e}$ excess in an almost pure $\nu_{\mu}$ neutrino beam would be major evidence for a non-vanishing $\theta_{13}$. But on the top of the statistical and systematic uncertainties, correlations and degeneracies between $\theta_{13}, \theta_{12}$, $\operatorname{sgn}\left(\Delta m_{31}^{2}\right)$, and the CP- $\delta$ phase degrade the accessible knowledge on $\theta_{13}$ [37]. Both reactor and accelerator programs will provide complementary results to better constrain the last undetermined parameters (see [38]).

In order to improve the Chooz results with reactor experiments, two (or more) identical detectors close to a power station are required. The first detector has to be located at a few hundred meters from the reactor cores to monitor the $\overline{\nu_{e}}$ flux and spectrum before the oscillations. The second detector has to be placed between 1 and $2 \mathrm{~km}$ away from the core, to search for a departure from the overall $1 / L^{2}$ behavior of the $\overline{\nu_{e}}$ energy spectrum, the footprint of oscillation [39]. At Chooz, the reactor induced systematic error was $1.9 \%$, but this class of uncertainties cancels with the new set up. Two identical detectors allow relative comparison, leading to a systematic error of $0.6 \%$, using standard technologies $[40,41]$. Of course, the statistical error has also to be decreased to a similar amount 3 .

Several sites have been proposed to host a new reactor experiment to search for $\theta_{13}$ : Angra dos Reis (Brazil), Braidwood, Diablo Canyon (US), Angra (Brazil), Chooz, Cruas, and Penly (France), Krasnoyarsk (Russia), Daya Bay (China), and Kashiwazaki (Japan). We present a selection of the current proposals [42]. We classified them into first generation experiments (see Figure 3), to be done in the near future, and second generation, more ambitious projects, which need a significant R\&D effort.

\subsubsection{First generation experiments}

The Double Chooz [40] experimental site is located close to the twin reactor cores of the Chooz nuclear power station, operated by the French company Electricité de France (EDF). The two, almost identical, detectors contain a 10 ton fiducial volume of liquid scintillator doped with $0.1 \%$ of Gadolinium (Gd). The underground laboratory of the first Chooz experiment, located $1.05 \mathrm{~km}$ (under $300 \mathrm{mwe}$ ) from the cores is going to be used again. The second detector will be installed at about $150 \mathrm{~m}$ from the nuclear cores. An artificial hill of about $20 \mathrm{~m}$ (60 mwe) height has to be erected above the detector. The detector design is an evolution of the Chooz detector (see Figure 4). Starting from the center of the target the detector elements are as follows: the neutrino target; a thick acrylic cylinder, filled with $0.1 \%$ Gd loaded liquid scintillator; the $\gamma$-catcher, filled with unloaded liquid scintillator (the role of this additional region is to determine the full positron energy, as well as most of the neutron energy released after neutron capture); a buffer region filled with non scintillating oil, to decrease the accidental backgrounds from PMTs radioactivity; the stainless steel structure supporting approximately 500 PMTs; a muon veto; an external shielding of steel protects the inner detector from the radioactivity of the rock; and finally an outer muon veto. The dominant error is the relative normalization between the two detectors. It is expected to be less than $0.6 \%$. Correlated events is the most severe background source. In total, the background rates (accidental + correlated) for the near detector would be the order of tens of events for 60 mwe overburden. For the far detector the total background is estimated between $1 / \mathrm{d}$ and $2 / \mathrm{d}$. This can be compared with the signal rates of $4,000 / \mathrm{d}$ and $80 / \mathrm{d}$ in 


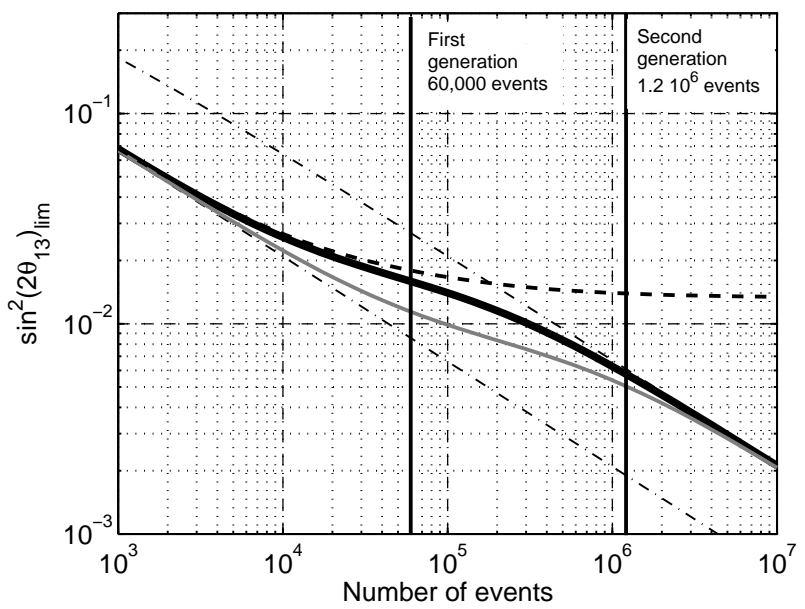

Figure 3. The sensitivity to $\sin ^{2}\left(2 \theta_{13}\right)$, at the $90 \%$ confidence level, as a function of the number of events in the far detector, for two different values of the relative normalization error: $0.6 \%$ (thick black curve) and $0.3 \%$ (thin gray curve). We considered a far detector located at $1.5 \mathrm{~km}$ from the cores, and a near detector close enough to measure the antineutrinos prior to their oscillation. We take the atmospheric mass splitting at $\Delta m_{a t m}^{2}=2.510^{-3} \mathrm{eV}^{2}$. The global normalization error is taken to be $2 \%$. The vertical lines mark the luminosities of the first and second generation experiments (60,000 and 1,200,000 events). The dashed line displays the sensitivity taking into account a $2 \%$ uncorrelated background subtraction error, in both cases; background will thus be crucial for the second generation of experiments [49].

the near and far detectors. The expected sensitivity is: $\sin ^{2}\left(2 \theta_{13}\right)<0.025$ (90\% C.L., for $\Delta m_{\text {atm }}^{2}=2.410^{-3} \mathrm{eV}^{2}$, for 3 years of operation) in the no-oscillation case. The discovery potential is around 0.04 ( 3 standard deviations). The Double Chooz collaboration plans to start taking data at the Chooz far/near site in 2007/2008.

Kaska is a Japanese collaboration aiming to start data taking end of 2008. Kaska [43] could be located close to the KAShiwazaki-KAriwa nuclear power station (BWR, $24.3 \mathrm{GW}_{\mathrm{th}}$ ). The plant is composed of 7 cores divided into 2 clusters spread by $2 \mathrm{~km}$. Thus, two near detectors are mandatory (each at $400 \mathrm{~m}$ from a cluster). The Kaska design is similar to the Double Chooz one : a 10 ton target of Gd doped liquid scintillator and a $\gamma$-catcher region enclosed in a double acrylic sphere, gamma shielding, a PMT supporting structure, and a weak scintillating region acting as a muon veto. The systematic error foreseen is between 0.5 and $1 \%$. The sensitivity is expected to be between $\sin ^{2}\left(2 \theta_{13}\right)<0.017-0.027\left(90 \%\right.$ C.L., for 3 years of operation, depending on the true value of $\left.\Delta m_{a t m}^{2}\right)$, in the no-oscillation case.

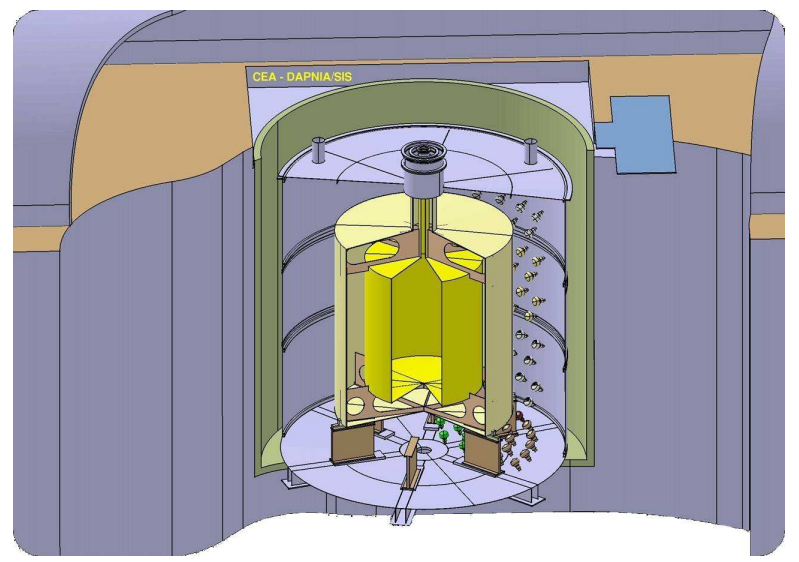

Figure 4. Overview of the Double Chooz detector (at the far site, $1.05 \mathrm{~km}$ from the nuclear cores). 


\subsubsection{Second generation experiments}

A second generation of experiments, with a large target mass, and ultra low and very well understood backgrounds, has been proposed in order to further improve the sensitivity to $\sin ^{2}\left(2 \theta_{13}\right)$. These experiments would be complementary to the lower limit's of sensitivity of the Superbeam program which probes the $\left(\theta_{13}, \delta\right.$-CP) plane $[44,38]$. The goal is to reach a sensitivity $\sin ^{2}\left(2 \theta_{13}\right)<0.01(90 \%$ C.L.).

The Daya Bay experiment [45] could be located in the Guang-Dong Province, close to the Daya Bay nuclear power plant (PWR, 11.6 $\mathrm{GW}_{\mathrm{th}}$ ). Since the power station has been built near a mountain, the plan is to excavate horizontal tunnels and several halls. This scheme would provide an excellent overburden for the detectors: 400 mwe at a distance of $300 \mathrm{~m}$ from the cores, and 1200 mwe at a distance of about $1.5-2.0 \mathrm{~km}$. This provides flexibility to optimize the detector rooms location according to the volatile best fitted $\Delta m_{a t m}$ value. The particularity of this experiment is to use the so-called "movable detector" concept. The three detectors could be swapped for cross calibration. The question of the systematic error introduced when moving such sensitive machines remains open, however.

The Braidwood experiment [46] could be located close to the Braidwood twin nuclear station (BWR, 7.2 $\mathrm{GW}_{\mathrm{th}}$ ), in Illinois. The area surrounding the power plant has a flat topology, thus two $120 \mathrm{~m}$ deep shafts as well as two large detector rooms have to be excavated. The overburden of 450 mwe would provide the same background contribution in each detector. Since all civil construction have to be realized, the detector locations could be optimized according to the true value of $\Delta m_{a t m}$. The plan is to have $\geq 1$ near detector of $25-50$ tons (fiducial mass) at $270 \mathrm{~m}$ in the near shaft, and $\geq 2$ far detectors identical to the near ones, at $\sim 1.8 \mathrm{~km}$ in the far shaft. The detector design omits the $\gamma$-catcher region, and thus differs significantly from the other proposals. As in Daya Bay the detectors could be swapped for cross calibration, but using a platform transporter as well as a high capacity crane for the operation, instead of a railway system.

The Angra experiment [47], near the $6 \mathrm{GW}_{\text {th }}$ power station of Angra dos Reis, is going to focus on a highluminosity approach to provide a full energy spectrum measurement of the oscillation signature. The far detector site could be located at $1.5 \mathrm{~km}$ from the primary reactor core, under $700 \mathrm{~m}$ of granite (1700 mwe). The detector would be a 500 ton fiducial volume of Gd loaded liquid scintillator. The near detector could be either identical to the far detector at $300 \mathrm{~m}$ from the core (covered by $100 \mathrm{~m}$ of granite), or smaller than the far detector (nonidentical) but very near to the nuclear core. If a luminosity of $6,000 \mathrm{GW}_{\mathrm{th}}$-ton-year can be achieved, the expected sensitivity is $\sin ^{2}\left(2 \theta_{13}\right)<0.007$.

\section{Neutrinos and society}

In the past, neutrino experiments have only been used for fundamental research, but today, thanks to the extraordinary progress of the field, e.g. the measurement of the oscillation parameters, neutrinos could be useful for Society. We will see that reactor experiments could play an important role in this new field in the near future.

\subsection{Non proliferation of nuclear weapons and reactor monitoring}

The International Atomic Energy Agency (IAEA) works with its Member States to promote safe, secure and peaceful nuclear technologies. One of its missions is to verify that safeguarded nuclear material and activities are not used for military purposes. In a context of international tension, neutrino detectors could help the IAEA to verify the Treaty on the Non-Proliferation of Nuclear Weapons (NPT), signed by 145 States around the world.

A small neutrino detector located at a few tens of meters from a nuclear core could monitor nuclear reactor cores non-intrusively, robustly and automatically. Since the antineutrino spectra and relative yields of fissioning isotopes ${ }^{235} \mathrm{U},{ }^{238} \mathrm{U},{ }^{239} \mathrm{Pu},{ }^{241} \mathrm{Pu}$ depend on the isotopic composition of the core, small changes in composition could be observed without ever directly accessing the core itself. Information from a modest-sized antineutrino detector, coupled with the well-understood principles that govern the core's evolution in time, can be used to determine whether the reactor is being operated in an illegitimate way (see Figure 1). Furthermore, such a detector can help to improve the reliability of the operation, by providing an independent and accurate measurement, in real time, of the thermal power and its reactivity at a level of a few percent. The intention is to design an "optimal" monitoring detector by using the experience obtained from neutrino physics experiments and feasibility studies.

Sands is a one cubic meter antineutrino detector located at 25 meters from the core of the San Onofre reactor site in California [48]. The detector has been operating for several months in an automatic and non-intrusive 
fashion that demonstrates the principles of reactor monitoring. Although the signal to noise ratio of the current design is still less than two, it is possible to monitor the thermal power at a level of a few percent in two weeks. At this stage of the work, the study of the evolution of the fuel seems difficult, but this has already been demonstrated by the Bugey and Rovno experiments [29,31].

Double Chooz will be a research detector with a very high sensitivity to study neutrino oscillations. About 20,000 neutrino events per year are expected in the far detector, but millions of events in the near detector (between 100 and $200 \mathrm{~m}$ away from the cores). These huge statistics could be exploited to help the IAEA in its Safeguards missions [40]. The potential of neutrinos to detect various reactor diversion scenario's can be tested with Double Chooz. A realistic reactor monitor is likely to be somewhere between the two concepts presented above.

\subsection{Geophysics}

The total measured heat dissipation rate from the Earth lies between 30 to $40 \mathrm{TW}$. Geo-neutrinos are the neutrinos and antineutrinos from the progenies of U, Th and $\mathrm{K}$ decays in the Earth, and Earth composition models suggest that about $20 \mathrm{TW}$ originate from these isotope decays (see for example [50] for more information on geo-neutrinos). U and Th end-point energies are above the threshold of inverse beta reactions on free protons, thus they can be detected as reactor antineutrinos. $\mathrm{U}$ and $\mathrm{Th}$ geoneutrinos could in principle be distinguished due to their different energy spectra, e.g. geo-neutrinos with energy $E>2.25 \mathrm{MeV}$ are produced only from the Uranium chain. In conclusion, antineutrinos could be used for determining the radiogenic contribution to the terrestrial heat flow and for discriminating among different models of the Earth's composition.

Since 2002 the KamLAND experiment has looked for geo-neutrinos and has just published updated data [51]. They have detected 4.5 to 54.2 geoneutrino events (90\% C.L.), whereas 19 events were predicted by the standard geophysical model. This provide, for the first time, a direct upper limit of $60 \mathrm{TW}$ (99\% C.L.) for the radiogenic power of $\mathrm{U}$ and $\mathrm{Th}$ in the Earth [51].

In the meanwhile, other projects for geo-neutrino detection are being planed. Borexino at Gran Sasso will benefit from the absence of nearby reactors. The LENA proposal [52] envisages a 50 kton liquid scintillator detector at the Center for Underground Physics in the Pyhasälmi mine (Finland). Due to the huge mass, it should collect several hundred events per year. In conclusion, one can expect that within 10 to 20 years the geo-neutrino signal from Uranium and Thorium will be measured at a few points on the globe.

A natural nuclear fission reactor with a power output of 3 to $10 \mathrm{TW}$ at the center of the Earth has been proposed as the energy source of the Earth's magnetic field [53]. The proposal can be directly tested by a massive liquid scintillator detector that can detect the signature spectrum of antineutrinos from the geo-reactor as well as the direction of the antineutrino source (Earth core). However, the clarity of both types of measurements may be limited by background from antineutrinos from surface power reactors.

\section{References}

[1] W. Pauli, Jr., Address to Group on Radioactivity (Tuebingen, December 4, 1930) (Unpublished) Septième conseil de physique Solvay, Bruxelles, 1033 (Gautier-Villars, Paris, France, 1934).

[2] E. Fermi, Z. Physik 88 (1934) 161.

[3] F. Reines and C.L. Jr. Cowan, Phys. Rev. 92 (1953) 830.

[4] C.L. Jr. Cowan, F. Reines, F.B. Harrison, H.W. Kruse and A.D. McGuire, Science 124 (1956) 103.

[5] F. Reines, C.L. Jr. Cowan, F.B. Harrison, A D. McGuire, H.W. and Kruse, Phys. Rev. 117 (1960) 159.

[6] F. Reines and C.L. Jr. Cowan, Phys. Rev. 113 (1959) 273.

[7] K. Schreckenbach et al., Phys. Lett. B160 (1985) 325; A. A. Hahn et al., Phys. Lett. B218 (1989) 365.

[8] B. Achkar et al., Phys. Lett. B374 (1996) 243.

[9] F. Reines, H.S. Gurr and H.W. Sobel, Phys. Rev. Lett. 37 (1976) 315; P. Vogel and J. Engel, Phys. Rev. D39 (1989) 3378.

[10] D.Z. Freedman et al., Annu. Rev. Nucl. Sci. 27 (1977) 167; J.R. Wilson, Phys. Rev. Lett. 32 (1974) 849.

[11] A. Drukier and L. Stodolsky, Phys. Rev. D30 (1984) 2295.

[12] B.W. Lee and R.E. Shrock, Phys. Rev. D16 (1977) 1444; W.J. Marciano and A.I. Sanda, Phys. Lett. B67 (1977) 303. 
[13] A.I. Derbin et al., JETP Lett. 57 (1993) 768.

[14] H.B. Li et al., Phys. Rev. Lett. 90 (2003) 131802.

[15] Z. Daraktchieva et al., Phys. Lett. B615 (2005) 153.

[16] K. Hagiwara et al., Particle Data Group, Phys. Rev. D66 (2002) 010001 and references therein.

[17] J.F. Beacom and P. Vogel, Phys. Rev. Lett. 83 (1999) 5222.

[18] T. Kajita and P. Lipari, this issue.

[19] M. Cribier and T. Bowles, this issue.

[20] Y. Fukuda et al., Phys. Rev. Lett. 81 (1998) 1562.

[21] S. Fukuda et al., Phys. Rev. Lett, 86 (2001) 5656; S. Fukuda et al., Phys. Lett. B539 (2002) 179.

[22] Q.R. Ahmad et al., Phys. Rev. Lett. 89 (2002) 011301; Q.R. Ahmad et al., Phys. Rev. Lett. 89 (2002) 011302.

[23] S.N. Ahmed et al., nucl-ex/0309004 (2004).

[24] K. Eguchi et al., Phys. Rev. Lett. 90 (2003) 021802.

[25] M.H. Ahn et al., Phys. Rev. Lett. 90 (2003) 041801.

[26] J. Bouchez, this issue.

[27] S. Schönert, Th. Lasserre and L. Oberauer, Astropart. Phys. 18 (2003) 565.

[28] G. Zacek et al., Phys. Rev. D34 (1986) 2621.

[29] A.F. Afonin et al., Sov. Phys. JETP Lett. 66 (1988) 213.

[30] G.S. Vidyakin et al., JETP Lett. 59 (1994) 390.

[31] B. Achkar et al., Nucl. Phys. B434 (1995) 503.

[32] Y. Déclais et al., Phys. Lett. B338 (1994) 383.

[33] M. Apollonio et al., Eur. Phys. J. C27 (2003) 331.

[34] T. Araki et al., Phys. Rev. Lett. 94 (2005) 081801.

[35] S. Schönert, Nucl. Phys. B (Proc. Suppl.) 70 (1999) 195.

[36] A. Bandyopadhyay, S. Choubey, S. Goswami and S.T. Petcov, hep-ph/0410283 (2004).

[37] P. Huber et al., Nucl. Phys. B665 (2003) 487.

[38] D. Autiero and Y. Déclais, this issue.

[39] K. Anderson et al., "White Paper For a New Reactor Neutrino Experiment for $\theta_{13}$ ", hep-ex/0402041 (2004).

[40] F. Ardellier et al. (Double-Chooz Collaboration), hep-ex/0405032 (2004).

[41] S. Berridge et al. (Double-Chooz Collaboration), hep-ex/0410081 (2004).

[42] Th. Lasserre, hep-ex/0411083 (2004).

[43] F. Suekane, hep-ex/0407016 (2004).

[44] H. Minakata et al., Phys. Lett. B580 (2004) 216.

[45] Q.Y. Liu, et al., hep-ph/0409155 (2004).

[46] Braidwood Reactor Experiment, http://mwtheta13.uchicago.edu/.

[47] D. Reyna, Third LENE Workshop, Niigata (2004), http://neutrino.hep.sc.niigata-u.ac.jp.

[48] A. Bernstein, "Neutrinos and arm control workshop", Hawaii (2003), http://www.phys.hawaii.edu/ jgl/nacw.

[49] G. Mention, Ph.D. thesis, Université Paris 7 (2005).

[50] G. Fiorentini et al., Nucl. Phys. B (Proc. Suppl.) 143 (2005) 53 ; G. Fiorentini, Th. Lasserre, M. Lissia, B. Ricci and S. Schönert, Phys. Lett. B558 (2003) 15.

[51] T. Araki et al., Nature 436 (2005) 499.

[52] L. Oberauer, Mod. Phys. Lett. A 19 (2004) 337.

[53] J.M. Herndon, Proc. Nat. Acad. Sci. U.S.A., 93(2) (1996) 646; ibidem 100(6) (2003) 3047. 